\title{
A Generalized Version of the Earle-Hamilton Fixed Point Theorem for the Hilbert Ball
}

\author{
David Shoikhet \\ Department of Mathematics, ORT Braude College \\ PO box 21982, Karmiel, Israel \\ Tel: 972-4-990-1861Ｅ-mail: davs@braude.ac.il
}

Received: December 26, 2011 Accepted: January 10, 2012 Published: April 1, 2012

doi:10.5539/jmr.v4n2p45 URL: http://dx.doi.org/10.5539/jmr.v4n2p45

\begin{abstract}
Let $D$ be a bounded domain in a complex Banach space. According to the Earle-Hamilton fixed point theorem, if a holomorphic mapping $F: D \mapsto D$ maps $D$ strictly into itself, then it has a unique fixed point and its iterates converge to this fixed point locally uniformly. Now let $\mathcal{B}$ be the open unit ball in a complex Hilbert space and let $F: \mathcal{B} \mapsto \mathcal{B}$ be holomorphic. We show that a similar conclusion holds even if the image $F(\mathcal{B})$ is not strictly inside $\mathcal{B}$, but is contained in a horosphere internally tangent to the boundary of $\mathcal{B}$. This geometric condition is equivalent to the fact that $F$ is asymptotically strongly nonexpansive with respect to the hyperbolic metric in $\mathcal{B}$.
\end{abstract}

Keywords: Fixed point, Hilbert space, Holomorphic self-mapping, Horosphere, Hyperbolic metric, Iterates, Local uniform convergence

\section{Introduction and Notions}

Let $\Delta$ be the open unit disk in the complex plane $\mathbb{C}$, and let $F$ be a holomorphic self-mapping of $\Delta$. Combining the classical Denjoy-Wolff Theorem and the Julia-Wolff-Carathéodory Theorem (Dineen, S., 1989; Cowen, C. C. et al., 1995; Goebel, K. et al., 1984; Shoikhet, D., 2001; Shapiro, J. H., 1993), it can be stated that:

$\checkmark$ If $F$ is not the identity and is not an elliptic automorphism of $\Delta$, then the iterates $F^{n}\left(=F \circ F^{n-1}\right), n=1,2, \ldots, F^{0}=I$, the identity mapping on $\Delta$, converge uniformly on the compact subsets of $\Delta$ to a constant mapping of $\Delta$ into $\bar{\Delta}$.

Moreover, the following are equivalent:

(i)F is fixed point free in $\Delta$ (that is there is no fixed point of $F$ in $\Delta$ ).

(ii)F has a boundary regular fixed point $\zeta\left(=\lim _{r \rightarrow 1^{-}} F(r \zeta)\right)$, such that $0<F^{\prime}(\zeta)\left(=\lim _{r \rightarrow 1^{-}} F^{\prime}(r \zeta)\right) \leq 1$.

(iii) There is a point $\eta \in \partial \Delta$ such that each horodisk internally tangent to $\partial \Delta$ at $\eta$ is $F$-invariant.

(iv) The sequence of iterates $\left\{F^{n}\right\}_{n=1}^{\infty}$ converges uniformly on the compact subsets of $\Delta$ to a boundary point $\tau \in \partial \Delta$.

The points $\zeta, \eta$ and $\tau$ in (ii)-(iv) must coincide.

Sometimes this assertion is called the Grand Fixed Point Theorem (see, for example, Shapiro, J. H., 1993). In general, it is no longer true for the infinite dimensional case. Various partial analogs of the Denjoy-Wolff and Julia-Wolff-Carathéodory Theorems for higher dimensions can be found in (Abate, 1998, p. 275-306; Abate et al., 1999, p. 161-172; Cowen, C. C. et al., 1995; Goebel, K. et al., 1984; Kuczumow et al., 2001, p. 437-515; MacCluer, 1983, p. 97-106; Reich et al., 1997, p. 219-240; Reich et al., 2002, p. 121-123; Shoikhet, D., 2001).

Let now $\mathcal{B}$ be the open unit ball in the complex Hilbert space $\mathcal{H}$, with the scalar product $\langle\cdot, \cdot\rangle$ and the norm $\|z\|=\sqrt{\langle z, z\rangle}$, $z \in \mathcal{H}$.

In this paper we study some geometric and analytic conditions which ensure the local uniform convergence of iterates of a holomorphic self-mapping of $\mathcal{B}$ to a constant mapping of the norm less or equal to 1 .

We need the following notions and facts.

Fix $a \in \mathcal{B}$. The Möbius transformation $m_{a}$ on $\mathcal{B}$ is defined by

$$
m_{a}(z)=\frac{1}{1+\langle z, a\rangle}\left(\sqrt{1-\|a\|^{2}} Q_{a}+P_{a}\right)(z+a)
$$

where $z \in \mathcal{B}, P_{a}$ is the orthogonal projection of $\mathcal{H}$ onto the subspace $\{\lambda a: \lambda \in \mathbb{C}\}$ and $Q_{a}=I-P_{a}, I$ is the identity 
mapping on $\mathcal{H}$.

The Poincaré hyperbolic metric on $\mathcal{B}$ is the function $\rho_{\mathcal{B}}: \mathcal{B} \times \mathcal{B} \rightarrow \mathbb{R}^{+}$given by

$$
\begin{aligned}
\rho_{\mathcal{B}}(z, w) & =\tanh ^{-1}\left\|m_{-z}(w)\right\|= \\
& =\frac{1}{2} \log \frac{1+\left\|m_{-z}(w)\right\|}{1-\left\|m_{-z}(w)\right\|}, \quad z, w \in \mathcal{B} .
\end{aligned}
$$

Note that $\rho_{\mathcal{B}}$ can be written in the form

$$
\rho_{\mathcal{B}}(z, w)=\tanh ^{-1} \sqrt{1-\sigma(z, w)}
$$

where

$$
\begin{aligned}
\sigma(z, w) & =1-\left\|m_{-z}(w)\right\|^{2}= \\
& =\frac{\left(1-\|z\|^{2}\right)\left(1-\|w\|^{2}\right)}{|1-\langle z, w\rangle|^{2}} .
\end{aligned}
$$

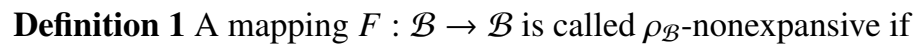

$$
\rho_{\mathcal{B}}(F(z), F(w)) \leq \rho_{\mathcal{B}}(z, w)
$$

for all $z, w \in \mathcal{B}$.

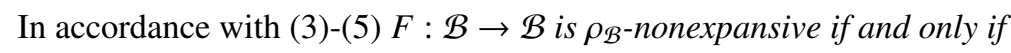

$$
\sigma(z, w) \leq \sigma(F(z), F(w))
$$

for all $z, w$ in $B$.

A consequence of the Schwarz-Pick Lemma is the fact that each holomorphic self-mapping of $\mathcal{B}$ is $\rho_{\mathcal{B}}$-nonexpansive (see, for example, Franzoni, T. et al., 1980; Goebel, K. et al., 1984; Reich, S. et al., 2005; Dineen, S., 1989).

Since $\mathcal{B}$ is a complete metric space with respect to the metric $\rho_{\mathcal{B}}$, the latter fact is very useful in the study of the fixed point sets of holomorphic self-mappings in $\mathcal{B}$ in the framework of the general fixed point theory on metric spaces.

At the same time, since $\rho_{\mathcal{B}}(z, w)$ goes to infinity if either $z$ or $w$ tends to the boundary $\partial \mathcal{B}$ of $\mathcal{B}$ little information can be derived about boundary behavior of holomorphic (or $\rho_{\mathcal{B}}$-nonexpansive) mappings even if they admit continuous extension onto $\partial \mathcal{B}$.

To avoid this deficiency, one induces another non-euclidean "distance" $d(z, w)$ from $z \in \mathcal{B}$ to $w \in \overline{\mathcal{B}}$, the closure of $\mathcal{B}$, defined by

$$
d(z, w)=\frac{|1-\langle z, w\rangle|^{2}}{1-\|z\|^{2}}
$$

(see, for example, Goebel, K. et al., 1984; Cowen, C. C. et al., 1995; Elin et al., 2002, p. 501-526).

Note that $d(z, w)$ is not a metric (it is even not symmetric for $z, w \in \mathcal{B},\|z\| \neq\|w\|$ ) on $\mathcal{B}$.

Geometrically the sets

$$
E(w, k)=\{z \in \mathcal{B}: d(z, w)<k\}, k>1-\|w\|^{2}, w \in \overline{\mathcal{B}},
$$

are ellipsoids in $\overline{\mathcal{B}}$ which for $w \in \partial \mathcal{B}$ are usually called horospheres.

Definition 2 Let $F$ be a continuous self-mapping of $\mathcal{B}$. A point $\zeta \in \partial \mathcal{B}$ is called a fixed point for $F$ if $\zeta=\lim _{r \rightarrow 1^{-}} F(r \zeta) \in$ $\partial \mathcal{B}$. It is called a boundary regular fixed point if the radial derivative

$$
\uparrow F^{\prime}(\zeta):=\lim _{r \rightarrow 1^{-}} \frac{1-\langle F(r \zeta), \zeta\rangle}{1-r}
$$

exists finitely.

We use the symbol $\uparrow F^{\prime}(\zeta)$ to distinguish the radial derivative (which is actually a positive real number, see (Cowen, C. C. et al., 1995; Reich, S. et al., 2005)) from the Frechét derivative usually denoted by $F^{\prime}(z)$ at the point $z \in \mathcal{B}$ which is a complex linear operator on $\mathcal{H}$ (see, for example, Franzoni, T. et al., 1980; Goebel, K. et al., 1984).

Definition 3 Let $F: \mathcal{B} \rightarrow \mathcal{B}$ be a $\rho_{\mathcal{B}}$-nonexpansive mapping on $\mathcal{B}$. A point $\tau \in \partial \mathcal{B}$ is called a sink point for $F$ if all ellipsoids $E(\tau, k), k>0$ are invariant for $F$. 
It can be shown (cf. Reich, S. et al., 2005, Theorems 5.14 and 5.15) that a point $\tau \in \partial B$ is a sink point of a $\rho_{\mathcal{B}^{-}}$ nonexpansive mapping $F$ on $B$ if and only if it is a boundary regular fixed point of $F$ with $\uparrow F^{\prime}(\zeta) \leq 1$.

A result of Goebel, Sekowski and Stachura (Goebel, K. et al., 1980, p. 1011-1021) (see also Goebel, K. et al., 1984, Theorem 25.2) asserts that:

$\checkmark$ If a $\rho$-nonexpansive (holomorphic) self-mapping $F: \mathcal{B} \rightarrow \mathcal{B}$ is fixed point free, then there is a unique sink point $\tau \in \partial \mathcal{B}$ for $F$.

In addition, B. MacCluer showed (MacCluer, 1983, p. 97-106), that if $\operatorname{dim} \mathcal{H}<\infty$, then iterates of a fixed point free holomorphic self-mapping $B$ converge uniformly on compact subsets on $\mathcal{B}$ to a sink point $\tau \in \partial \mathcal{B}$.

However, if $\operatorname{dim} \mathcal{H}>1$, then even for holomorphic mappings, a converse assertion is no longer true: if $F$ has a boundary sink point, then $F$ is not necessarily fixed point free (see Theorem 25.1 in Goebel, K. et al., 1984 and examples therein).

Moreover, in contrast with the finite-dimensional case an example of A. Stachura (Stachura, 1985, p. 88-90) shows that for the infinite dimensional case iterates of a holomorphic self-mapping of $\mathcal{B}$ do not necessarily converge to a sink point even if it is a unique boundary regular fixed point of $F$.

On the other hand, one can show (see the proof of Theorem 30.8 in Goebel, K. et al., 1984) that if a $\rho_{\mathcal{B}}$-nonexpansive mapping $F$ in $\mathcal{B}$ is fixed point free and $\tau$ is its sink point then the inequality

$$
d(F(z), \tau) \leq d\left(\frac{1}{2}(z+F(z)), \tau\right)
$$

provides the pointwise convergence (in the norm of $\mathcal{H}$ ) of iterates $F^{n}(z)$ to the point $\tau$ for all $z \in \mathcal{B}$.

\section{Local Uniform Convergence of Iterates}

Even though the strong and weak convergence of iterates has been studied very intensively (Goebel et al., 1982, p. 349352; Reich, 1985, p. 199-206), see also book (Goebel, K. et al., 1984) and survey (Kuczumow et al., 2001, p. 437-515 and references therein), little is known about local uniform convergence of iterates for the infinite dimensional case. Some results concerning this issue for compact or condensing holomorphic mappings can be found in (Kapeluszny et al., 1999, p. 111-123; Kapeluszny et al., 1999, p. 79-93).

Definition 4 (cf. Franzoni, T. et al., 1980; Reich, S. et al., 2005) We say that a subset $K$ of a bounded domain $\mathcal{D}$ in a Banach space $X$ is strictly inside $\mathcal{D}$, if it is bounded away from the boundary of $\mathcal{D}$, i.e., inf ${ }_{z \in \mathcal{K}}\|z-w\| \geq \varepsilon>0$.

A sequence $\left\{F^{n}\right\}_{n=1}^{\infty}$ of mappings on $\mathcal{D}$ is said to be locally uniformly convergent on $D$ if it converges uniformly (in the norm of $X$ ) on each ball strictly inside $\mathcal{D}$.

The famous Earle-Hamilton Theorem (Earle et al., 1970, p. 61-65) (see also Dineen, S., 1989; Goebel, K. et al., 1984; Harris, 2003, p. 261-274; Reich, S. et al., 2005) asserts that:

$\checkmark$ Let $F$ be a holomorphic self-mapping of a bounded domain D in a complex Banach space X. If F maps D into a subset $K$ strictly inside $D$, then $F$ has a unique fixed point $\zeta \in D$ and iterates $\left\{F^{n}\right\}_{n=1}^{\infty}$ converge to $\zeta$ locally uniformly in D.

For $\mathbb{C}^{n}$ this result was partially established earlier by M.Herve (Hervé, M., 1963) as a consequence of the Remmert-Stein Theorem.

In the setting of the Hilbert ball we show that a similar conclusion holds even if the image $F(\mathcal{B})$ is not strictly inside $\mathcal{B}$, but belongs to a horosphere in $\overline{\mathcal{B}}$, the closure of $\mathcal{B}$.

To formulate our result we need some additional notations.

For a linear operator $A: X \mapsto X$, we denote by $\Sigma(A)$ the spectrum of $A$, and by $\Sigma_{p}(A)=\{\lambda \in \Sigma(A): \lambda$ is an eigen-value of $A$ ) the point spectrum of $A$.

By $\Sigma_{\partial \Delta}(A)=\{\lambda \in \Sigma(A),|\lambda|=1\}$ we denote the peripheral spectrum of $A$, whenever, $\Sigma(A) \subseteq \bar{\Delta}$, the closure of the unit disk.

It is well known that if a holomorphic self-mapping $F$ of a bounded domain $D$ in a Banach space $X$ has a fixed point $\zeta \in D$, then $\Sigma\left(F^{\prime}(\zeta)\right) \subseteq \bar{\Delta}$ (see, for example, Vesentini, 1985, p. 13-16; Reich, S. et al., 2005).

Now we formulate our main result.

Theorem 5 Let $F$ be a holomorphic mapping on $\mathcal{B}$ which maps $\mathcal{B}$ into a horosphere $E(\tau, m)$ for some $\tau \in \partial \mathcal{B}$ and $0<m<\infty$, i.e.,

$$
d(F(z), \tau)<m<\infty, \quad z \in \mathcal{B} .
$$

The following assertions hold. 
(i) F has at most one fixed point in $\mathcal{B}$.

(ii) If $F(\zeta)=\zeta \in \mathcal{B}$ and $A=F^{\prime}(\zeta)$ has the peripheral spectrum $\Sigma_{\partial \Delta}(A)$ which belongs to the point spectrum $\Sigma_{p}(A)$, then the iterates $\left\{F^{n}\right\}_{n=1}^{\infty}$ converge to the point $\zeta$ in the topology of local uniform convergence on $\mathcal{B}$.

(iii) If $F$ has a boundary regular fixed point, then it must be $\tau$.

(iv) $F$ is fixed point free if and only if $\tau(\in \partial \mathcal{B})$ is a sink point for $F$ and if and only if iterates $\left\{F^{n}\right\}_{n=1}^{\infty}$ converge to the point $\tau$ in the topology of the local uniform convergence on $\mathcal{B}$.

Remark 6 It follows from the results in (Lyubich et al., 1994, p. 89-97) (see, also Kaashek et al., 1968, p. 428-438; Świȩch, 1991, p. 277-282) that a power bounded linear operator $A$ satisfies condition (ii) of the theorem if and only if it is uniformly precompact (in the norm operator topology). This fact and the Cauchy inequalities for the Frechét derivatives (see, for example, Franzoni, T. et al., 1980; Goebel, K. et al., 1984; Reich, S. et al., 2005) imply the following:

Corollary 7 Let $F$ be a holomorphic mapping on $\mathcal{B}$ which satisfies (8). Then the sequence $\left\{F^{n}\right\}_{n=1}^{\infty}$ of iterates locally uniformly converges to a constant mapping in $\overline{\mathcal{B}}$ if and only if it is locally uniformly precompact.

In particular, if $F(\mathcal{B})$ is relatively compact, then the sequence $\left\{F^{n}\right\}_{n=1}^{\infty}$ always converges to a fixed point of $F$ in $\mathcal{B}$ in the open compact topology on $\mathcal{B}$.

It should be observed that the key point in the proof of the Earle-Hamilton Theorem is the fact that each holomorphic self mapping of $D$ which maps $D$ into a subset $K$ strictly inside $D$ is a strict contraction with respect to a hyperbolic metric on D. So, the Banach Fixed Point Principle can be applied.

\section{Asymptotically Strongly Nonexpansive Mappings}

To prove our main theorem we first establish another metric characterization of holomorphic mappings which satisfy condition (8).

Definition 8 We say that a mapping $F: \mathcal{B} \rightarrow \mathcal{B}$ is asymptotically strongly nonexpansive with respect to a point $\tau \in \partial \mathcal{B}$ if there is a positive function $p_{F}$ on $\mathcal{B} \times \mathcal{B}$ such that

(a) $0 \leq p_{F}(z, w) \leq 1, \quad p_{F}(z, w) \not \equiv 0, \quad z, w \in \mathcal{B}$;

(b) $\lim _{r \rightarrow 1^{-}} \frac{p_{F}(z, r \tau)}{1-r}:=k>0$ for all $z \in \mathcal{B}$;

and

(c) for all $z, w \in \mathcal{B}$,

$$
\sigma(F(z), F(w)) \geq \sigma(z, w)\left(1-p_{F}(z, w)\right)+p_{F}(z, w)(\geq \sigma(z, w)) .
$$

We remark in passing that another class of strongly nonexpansive mappings in the Hilbert ball was considered in (Reich et al., 1993, p. 21-25; Kopecká, E. et al., 2009, p. 3187-3194).

Theorem 9 Let $F$ be a holomorphic self-mapping of $\mathcal{B}$, such that for some $\tau \in \partial \mathcal{B}, \lim _{r \rightarrow 1^{-}} F(r \tau)=\tau$ and the radial derivative $\uparrow F^{\prime}(\tau)$ exists finitely. Then $F$ is asymptotically strongly nonexpansive with respect to $\tau$ if and only if there is a positive number $m<\infty$ such that condition (8) holds.

Moreover,

$$
m \geq \frac{2\left(\uparrow F^{\prime}(\tau)\right)}{k},
$$

where $k$ is defined by condition (b) of Definition 8.

Proof: Assume that (8) holds. For $x \in \mathcal{H}$ define

$$
S(x)=\mathfrak{R}\langle x, \tau\rangle+|\langle x, \tau\rangle|^{2}-\|x\|^{2}
$$

and let $\Pi\left(=\Pi_{\tau}\right):=\{x \in \mathcal{H}: S(x)>0\}$ be Siegel's domain in $\mathcal{H}$. So, if

$$
C(z)\left(=C_{\tau}(z)\right):=\frac{1}{1-\langle z, \tau\rangle}(z+\tau)
$$

is the Caley transformation of $\mathcal{B}$, then $C(\mathcal{B})=\Pi$ and $C^{-1}(\Pi)=\mathcal{B}$. 
In addition, for each $z \in \mathcal{B}$ we have

$$
\begin{aligned}
S(C(z)) & =\mathfrak{R}\langle C(z), \tau\rangle+|\langle C(z), \tau\rangle|^{2}-\|C(z)\|^{2}= \\
& =\mathfrak{R} \frac{\langle z, \tau\rangle+1}{1-\langle z, \tau\rangle}+\frac{|1+\langle z, \tau\rangle|^{2}}{|1-\langle z, \tau\rangle|^{2}}-\frac{\|z\|^{2}+1+2 \mathfrak{R}\langle z, \tau\rangle}{|1-\langle z, \tau\rangle|^{2}} \\
& =\frac{1-\|z\|^{2}}{|1-\langle z, \tau\rangle|^{2}}=\frac{1}{d(z, \tau)} .
\end{aligned}
$$

Therefore, condition (8) can be rewritten in the form

$$
S(C(F(z)))>\frac{1}{m}=: a>0 .
$$

On the other hand, since by (10)

$$
S(x-a \tau)=\mathfrak{R}\langle x, \tau\rangle-a+|\langle x, \tau\rangle-a|^{2}-\|x-a \tau\|^{2}=S(x)-a,
$$

it follows from (13) that the mapping $F_{1}$ :

$$
F_{1}(z)=C^{-1}(C(F(z))-a \tau)
$$

is well defined holomorphic mapping on $\mathcal{B}$ and maps $\mathcal{B}$ into itself.

Therefore, we get that $F_{1}$ is $\rho_{\mathcal{B}}$-nonexpansive on $\mathcal{B}$ which can be expressed by the inequality

$$
\sigma\left(F_{1}(z), F_{1}(w)\right) \geq \sigma(z, w)
$$

for all $z, w \in \mathcal{B}$.

Furthermore, setting $x=C(z)$ and $y=C(w)$ one calculates that

$$
\sigma(z, w)=\frac{4 S(x) S(y)}{|T(x, y)|^{2}}
$$

where

$$
T(x, y)=\langle x, \tau\rangle+\langle\tau, y\rangle+2(\langle x, \tau\rangle\langle\tau, y\rangle-\langle x, y\rangle) .
$$

In addition, it follows from (18) that

$$
T(x-a \tau, y-a \tau)=T(x, y)-2 a .
$$

If we set now $x_{1}=x-a \tau, x=C(F(z))$ and $y_{1}=y-a \tau, y=C(F(w))$ then we have by (14)-(19)

$$
\begin{aligned}
\sigma\left(F_{1}(z), F_{1}(w)\right) & =\frac{4 S\left(x_{1}\right) \cdot S\left(y_{1}\right)}{\left|T\left(x_{1}, y_{1}\right)\right|^{2}}=\frac{4 S(x-a \tau) \cdot S(y-a \tau)}{|T(x-a \tau, y-a \tau)|^{2}} \\
& =\frac{4(S(x)-a) \cdot(S(y)-a)}{|T(x, y)-2 a|^{2}} \\
& =\frac{4 S(x) S(y)-4 a[S(x)+S(y)]+4 a^{2}}{|T(x, y)|^{2}-4 a \mathfrak{R} T(x, y)+4 a^{2}} .
\end{aligned}
$$

Then by using (17), (20) we obtain

$$
\sigma\left(F_{1}(z), F_{1}(w)\right)=\frac{\sigma(F(z), F(w))-b_{F}(z, w)}{1-c_{F}(z, w)},
$$

where we denote

$$
b_{F}(z, w)=\frac{4 a[S(C(F(z)))+S(C(F(w)))-a]}{|T(C(F(z)), C(F(w)))|^{2}}
$$

and

$$
c_{F}(z, w)=\frac{4 a \mathfrak{R}[T(C(F(z)), C(F(w)))-a]}{|T(C(F(z)), C(F(w)))|^{2}} .
$$

Since $S(C(F(z))) \geq a$, it is clear that $b_{F}(z, w)>0$. 
Now we prove that

$$
0<b_{F}(z, w) \leq c_{F}(z, w)<1, \quad z, w \in \mathcal{B} .
$$

To do this we have to show that

$$
\mathfrak{R} T(x, y) \geq S(x)+S(y)
$$

and

$$
4 a(\mathfrak{R} T(x, y)-a) \leq|T(x, y)|^{2},
$$

whenever $\min (S(x), S(y)) \geq a$, for $x, y \in \Pi$.

Indeed, by (18)

$$
\mathfrak{R} T(x, y)=\mathfrak{R}[\langle x, \tau\rangle+\langle\tau, y\rangle+2(\langle x, \tau\rangle\langle\tau, y\rangle-\langle x, y\rangle)] .
$$

Hence, by (10) inequality (25) can be rewritten as

$$
2 \mathfrak{R}(\langle x, \tau\rangle\langle\tau, y\rangle-\langle x, y\rangle) \geq|\langle x, \tau\rangle|^{2}+|\langle y, \tau\rangle|^{2}-\|x\|^{2}-\|y\|^{2}
$$

or

$$
\|x-y\|^{2} \geq|\langle x-y, \tau\rangle|^{2}
$$

which is obvious.

Note that, in fact, (27) holds for all $x, y \in \mathcal{H}$, so does (25). Inequality (26) is also evident since in view of (25) and (13) we have that

$$
|T(x, y)|^{2}-4 a \Re T(x, y)+4 a^{2}=|T(x, y)-2 a|^{2} \geq[S(x)+S(y)-2 a]^{2}>0 .
$$

Thus, relation (24) is proved.

Furthermore, we calculate by using (18) and (11)

$$
\begin{aligned}
T(C(F(z)), C(F(w)))=\langle C(F(z)), \tau\rangle+\langle\tau, C(F(w))\rangle \\
\quad+2(\langle C(F(z)), \tau\rangle\langle\tau, C(F(w))\rangle-\langle C(F(z)), C(F(w))\rangle) \\
\quad=\frac{1}{1-\langle\tau, F(w)\rangle} \cdot\left[\frac{1+\langle F(z), \tau\rangle}{1-\langle F(z), \tau\rangle} \cdot(1-\langle\tau, F(w)\rangle)+1+\langle\tau, F(w)\rangle\right. \\
\quad+2\left(\frac{1+\langle F(z), \tau\rangle}{1-\langle F(z), \tau\rangle}(1+\langle\tau, F(w)\rangle)\right. \\
\left.\left.\quad-\left\langle\frac{1}{1-\langle F(z), \tau\rangle}(F(z)+\tau), F(w)+\tau\right\rangle\right)\right]
\end{aligned}
$$

Consider now the following function

$$
A(r)=(1-r)^{2}|T(C(F(z)), C(F(r \tau)))|^{2} .
$$

Since

$$
\lim _{r \rightarrow 1^{-}} F(r \tau)=\tau
$$

and

$$
\lim _{r \rightarrow 1^{-}} \frac{|1-\langle\tau, F(r \tau)\rangle|}{1-r}=\lim _{r \rightarrow 1^{-}} \frac{1-\|F(r \tau)\|}{1-r}=\uparrow F^{\prime}(\tau),
$$

(see Theorems 5.12 - 5.14 in Reich, S. et al., 2005) we obtain from (28)

$$
\begin{aligned}
\lim _{r \rightarrow 1^{-}} A(r) & =\lim _{r \rightarrow 1^{-}} \frac{(1-r)^{2}}{|1-\langle\tau, F(r \tau)\rangle|^{2}} \cdot \lim _{r \rightarrow 1^{-}} \mid \frac{1+\langle F(z), \tau\rangle}{1-\langle F(z), \tau\rangle} . \\
& (1-\langle\tau, F(r \tau)\rangle)+1+\langle\tau, F(r \tau)\rangle+2\left(\frac{1+\langle F(z), \tau\rangle}{1-\langle F(z), \tau\rangle} .\right. \\
& \left.(1+\langle\tau, F(r \tau)\rangle)-\left\langle\frac{F(z)+\tau}{1-\langle F(z), \tau\rangle}, F(r \tau)+\tau\right\rangle\right) \mid \\
& =4 \lim _{r \rightarrow 1^{-}} \frac{(1-r)^{2}}{|1-\langle\tau, F(r \tau)\rangle|^{2}}=\frac{4}{\left(\uparrow F^{\prime}(\tau)\right)^{2}} .
\end{aligned}
$$


Hence,

$$
\lim _{r \rightarrow 1^{-}}(1-r)|T(C(F(z)), C(F(r \tau)))|^{2}=\lim _{r \rightarrow 1^{-}} \frac{A(r)}{1-r}=\infty .
$$

Then we obtain from (22) and (28)-(31):

$$
\begin{aligned}
\lim _{r \rightarrow 1^{-}} \frac{b_{F}(z, r \tau)}{1-r} & =\lim _{r \rightarrow 1^{-}} \frac{4 a[S(C(F(z)))+S(C(F(r \tau)))-a]}{(1-r) \mid T\left(C(F(z)),\left.C(F(r \tau))\right|^{2}\right.} \\
& =\lim _{r \rightarrow 1^{-}} \frac{4 a(1-r)[S(C(F(z)))+S(C(F(r \tau)))-a]}{A(r)} \\
& =a \cdot\left(\uparrow F^{\prime}(\tau)\right)^{2} \cdot \lim _{r \rightarrow 1^{-}}(1-r) S(C(F(r \tau))) \\
& =a \cdot\left(\uparrow F^{\prime}(\tau)\right)^{2} \lim _{r \rightarrow 1^{-}} \frac{(1-r)\left(1-\|F(r \tau)\|^{2}\right)}{|1-\langle F(r \tau), \tau\rangle|^{2}} \\
& =2 a\left(\uparrow F^{\prime}(\tau)\right)=\frac{2\left(\uparrow F^{\prime}(\tau)\right)}{m}>0 .
\end{aligned}
$$

Since

we also get from (23) and (31)

$$
\frac{\mathfrak{R} T(x, y)}{|T(x, y)|} \leq 1
$$

$$
\begin{aligned}
0 & \leq \lim _{r \rightarrow 1^{-}} c_{F}(z, r \tau) \leq \\
& \leq 4 a \lim _{r \rightarrow 1^{-}}\left[\frac{1}{|T(C(F(z)), C(F(r \tau)))|}-\frac{a}{|T(C(F(z)), C(F(r \tau)))|^{2}}\right]=0 .
\end{aligned}
$$

To proceed, we need the following observation:

If $\rho_{\mathcal{B}}(\cdot, \cdot)$ is the Poincaré hyperbolic metric on $\mathcal{B}$, then one can define the hyperbolic metric on $\Pi=C(\mathcal{B})$ by the equality

$$
\rho_{\Pi}(x, y)=\rho_{\mathcal{B}}\left(C^{-1}(x), C^{-1}(y)\right),
$$

whenever, $x$ and $y$ are in $\Pi$. Since the affine mapping $h$ defined by

$$
h(x)=x+a \tau
$$

maps $\Pi$ into itself we have that

$$
\rho_{\Pi}(x+a \tau, y+a \tau) \leq \rho_{\Pi}(x, y)
$$

or

$$
\rho_{\mathcal{B}}\left(C^{-1}(x+a \tau), C^{-1}(y+a \tau)\right) \leq \rho_{\mathcal{B}}\left(C^{-1}(x), C^{-1}(y)\right), \quad x, y \in \Pi .
$$

Since $S(C(F(z))-a \tau)=S(C(F(z)))-a>0$ and $S(C(F(w))-a \tau)=S(C(F(w)))-a>0$ for all $z, w \in \mathcal{B}$, one can set $x=C(F(z))-a \tau$ and $y=C(F(w))-a \tau$ to obtain

$$
\rho_{\mathcal{B}}(F(z), F(w)) \leq \rho_{\mathcal{B}}\left(F_{1}(z), F_{1}(w)\right)
$$

which is equivalent to

$$
\sigma(F(z), F(w)) \geq \sigma\left(F_{1}(z), F_{1}(w)\right)=\frac{\sigma(F(z), F(w))-b_{F}(z, w)}{1-c_{F}(z, w)} .
$$

This implies that

$$
\sigma(z, w) \leq \sigma(F(z), F(w)) \leq \frac{b_{F}(z, w)}{c_{F}(z, w)} \leq 1 .
$$

In turn, the last inequality together with (16) and (21) implies that

$$
\sigma(F(z), F(w)) \geq\left(1-c_{F}(z, w)\right) \sigma(z, w)+b_{F}(z, w) \geq \sigma(z, w) .
$$

Now let us define a real function $p_{F}: \mathcal{B} \times \mathcal{B} \mapsto \mathbb{R}$ by the formula

$$
p_{F}(z, w)=\frac{b_{F}(z, w)-\sigma(z, w) c_{F}(z, w)}{1-\sigma(z, w)} .
$$


It follows from (34) that

$$
p_{F}(z, w) \geq 0, \quad z, w \in \mathcal{B}, \quad z \neq w .
$$

Also, since

$$
\lim _{r \rightarrow 1^{-}} \frac{\sigma(z, r \tau)}{1-r}=\lim _{r \rightarrow 1^{-}} \frac{\left(1-\|z\|^{2}\right)\left(1-r^{2}\right)}{|1-\langle z, r \tau\rangle|^{2}(1-r)}=\frac{2}{d(z, \tau)}<\infty,
$$

we obtain from (32) and (33)

$$
\begin{aligned}
k: & =\lim _{r \rightarrow 1^{-}} \frac{p_{F}(z, r \tau)}{1-r}=\lim _{r \rightarrow 1^{-}} \frac{\frac{1}{1-r}\left(b_{F}(z, r \tau)-\sigma(z, r \tau) c_{F}(z, r \tau)\right)}{1-\sigma(z, r \tau)} \\
& =\lim _{r \rightarrow 1^{-}} \frac{b_{F}(z, r \tau)}{1-r}=\frac{2\left(\uparrow F^{\prime}(\tau)\right)}{m}>0 .
\end{aligned}
$$

Finally, we have from (36) the equality

$$
\left(1-p_{F}(z, w)\right) \sigma(z, w)+p_{F}(z, w)=\left(1-c_{F}(z, w)\right) \sigma(z, w)+b_{F}(z, w),
$$

which proves together with (35) the inequality $p_{F}(z, w) \leq 1$ and condition (c) of Definition 8 .

This completes the proof of the sufficient part of the theorem.

To prove the converse part of Theorem 9 we establish a more general assertion which holds actually for asymptotically strongly nonexpansive mappings which are not necessarily holomorphic. This assertion also proves items (iii) and (iv) of Theorem 5 .

Theorem 10 Let $F: \mathcal{B} \rightarrow \mathcal{B}$ be an asymptotically strongly nonexpansive with respect to a point $\tau \in \partial \mathcal{B}$ (see Definition 8). Assume that $\tau$ is a boundary regular fixed point of $F$, i.e., $\lim _{r \rightarrow 1^{-}} F(r \tau)=\tau$ and the radial derivative $\uparrow F^{\prime}(\tau)=\beta>0$ exists finitely. Then for all $z \in \mathcal{B}$ the following inequality holds

$$
\frac{1}{d(F(z), \tau)} \geq \frac{1}{\beta}\left(\frac{k}{2}+\frac{1}{d(z, \tau)}\right)
$$

where $k=\lim _{r \rightarrow 1^{-}} \frac{p_{F}(z, r \tau)}{1-r}$.

If $F$ has a boundary regular fixed point then it must be $\tau$. In particular, the following are equivalent:

(i) The mapping $F$ is fixed point free.

(ii) The point $\tau$ is a sink point for $F$.

(iii) The sequence of iterates $\left\{F^{n}\right\}_{n=1}^{\infty}$ converges to the point $\tau$ locally uniformly on $\mathcal{B}$.

Moreover, the following rate of convergence holds

$$
d\left(F^{n}(z), \tau\right) \leq \alpha(n, z) \cdot d(z, \tau)
$$

where

$$
\alpha(n, z)=\left\{\begin{array}{c}
\frac{2}{2+n k d(z, \tau)}, \text { if } \beta=1 \\
\frac{2 \beta^{n}}{2(1-\beta)+\left(1-\beta^{n}\right) k}, \text { if } \beta<1
\end{array} .\right.
$$

Proof: Let $F$ be a holomorphic self-mapping which satisfies conditions (a)-(c) of Definition 8 .

Then we have

$$
\begin{aligned}
\frac{\left(1-\|F(z)\|^{2}\right)\left(1-\|F(r \tau)\|^{2}\right)}{|1-\langle F(z), F(r \tau)\rangle|^{2}} & \geq \\
& \geq\left(1-p_{F}(z, r \tau)\right) \frac{\left(1-\|z\|^{2}\right)\left(1-r^{2}\right)}{|1-\langle z, r \tau\rangle|^{2}}+p_{F}(z, r \tau)
\end{aligned}
$$


or

$$
\begin{aligned}
\frac{1-\|F(z)\|^{2}}{|1-\langle F(z), F(r \tau)\rangle|^{2}} \cdot \frac{1-\|F(r \tau)\|^{2}}{1-r^{2}} & \geq \\
& \geq\left(1-p_{F}(z, r \tau)\right) \frac{1-\|z\|^{2}}{|1-\langle z, r \tau\rangle|^{2}}+\frac{p_{F}(z, r \tau)}{1-r^{2}} .
\end{aligned}
$$

Letting $r \rightarrow 1^{-}$we get

$$
\frac{1}{d(F(z), \tau)} \geq \frac{1}{\beta}\left(\frac{k}{2}+\frac{1}{d(z, \tau)}\right)
$$

Let us assume, that there is a boundary regular fixed point $\eta$ of $F$, i.e., the radial derivative $L=\uparrow F^{\prime}(\eta)$ exists finitely.

Then we have by Theorem 5.12 in (Reich, S. et al., 2005)

$$
d(F(z), \eta)=\frac{|1-\langle F(z), \eta\rangle|^{2}}{1-\|F(z)\|^{2}} \leq L \frac{|1-\langle z, \eta\rangle|^{2}}{1-\|z\|^{2}}=L d(z, \eta) .
$$

Choose any $\varepsilon>0$ and element $z \in \mathcal{B}$ such that $L d(z, \eta)<\varepsilon^{2}$. Setting $w=F(z)$ we have that

$$
|1-\langle w, \eta\rangle|^{2}<\varepsilon^{2}
$$

and

$$
1-\|w\|^{2} \leq 1-|\langle w, \eta\rangle|^{2} \leq 2|1-\langle w, \eta\rangle|<2 \varepsilon
$$

Also, note that

$$
1-\mathfrak{R}\langle w, \eta\rangle\langle|1-\langle w, \eta\rangle|<\varepsilon .
$$

On the other hand, since $d(w, \tau)<m$, inequality (38) implies that

$$
|1-\langle w, \tau\rangle|^{2}<m\left(1-\|w\|^{2}\right) \leq 2 m \varepsilon .
$$

Hence, again

$$
1-\mathfrak{R}\langle w, \tau\rangle \leq|1-\langle w, \tau\rangle| \leq \delta=\sqrt{2 m \varepsilon} .
$$

Then we obtain

$$
\begin{aligned}
\|\eta-\tau\|^{2} & \leq(\|\eta-w\|+\|\tau-w\|)^{2} \leq \\
& \leq 2\left(\|\eta-w\|^{2}+\|\tau-w\|^{2}\right)= \\
& =4\left(1-\mathfrak{R}(\langle\eta, w\rangle+\langle\tau, w\rangle)+\|w\|^{2}\right) \\
& <4(1-\mathfrak{R}\langle\eta, w\rangle+1-\mathfrak{R}\langle\tau, w\rangle) \\
& <4(\varepsilon+\delta) .
\end{aligned}
$$

Since $\delta \rightarrow 0^{+}$as $\varepsilon \rightarrow 0^{+}$we have that $\tau=\eta$.

It is now clear that if $F$ is fixed point free, then a sink point $\eta \in \partial \mathcal{B}$ for $F$ (which is a boundary regular fixed point) must be $\tau$.

Let now $\tau \in \partial \mathcal{B}$ be a sink point for $F$. In this case $\beta \leq 1$ and we have by induction from inequality (37) that

$$
\begin{aligned}
& \frac{1}{d\left(F^{n}(z), \tau\right)} \geq \frac{1}{\beta^{n}} \frac{1}{d(z, \tau)}+\frac{k}{2 \beta}\left(1+\frac{1}{\beta}+\ldots+\frac{1}{\beta^{n-1}}\right)= \\
& \frac{1}{d(z, \tau)}+\frac{n k}{2}, \text { if } \beta=F^{\prime}(\tau)=1 \\
& \\
&=\left\{\begin{array}{c}
\frac{1}{\beta^{n}} \cdot\left[\frac{1}{d(z, \tau)}+\frac{1-\beta^{n}}{1-\beta} \frac{k}{2}\right], \quad \text { if } \beta=F^{\prime}(\tau)<1 .
\end{array}\right.
\end{aligned}
$$

This proves the implication (ii) $\Rightarrow$ (iii). The implication (iii) $\Rightarrow$ (i) is obvious, and we are done. 


\section{Proof of Main Result}

Proof of Theorem 5: (i) If $F$ has more than one fixed point in $\mathcal{B}$, then it follows from Rudin's Theorem (see Rudin, W., 1980 ), that the fixed point set $\mathcal{F}=F i x_{\mathcal{B}} F$ is an affine subset of $\mathcal{B}$, hence there is $x \in \mathcal{F}$, such that $x(=F(x)) \notin E(\tau, m)$. A contradiction.

(ii) Suppose that $\mathcal{F} \neq \varnothing$, i.e., there is $\zeta \in \mathcal{B}$ such that $\zeta=F(\zeta)$.

Assume also that $A=F^{\prime}(\zeta)$ satisfies the condition

$$
\Sigma_{\partial \Delta}(A) \subseteq \Sigma_{p}(A)
$$

First we show that, in fact, under our assumptions the peripheral spectrum $\Sigma_{\partial \Delta}(A)$ is empty.

Indeed, assume on the contrary that for some $\ominus \in[0,2 \pi]$ there is $x \neq 0$, such that

$$
e^{i \ominus} x=A x
$$

Consider a holomorphic self-mapping $F_{1}$ of $\mathcal{B}$ defined as follows

$$
F_{1}=e^{-i \ominus} \Phi \circ F \circ \Phi^{-1}
$$

where $\Phi:=m_{-\zeta}$ is the Möbius transformation of $\mathcal{B}$ defined by $(1)$, taking the point $\zeta$ to the origin $(\Phi(\zeta)=0)$. Then $F_{1}(0)=0$ and, by the chain rule

$$
A_{1}:=F_{1}^{\prime}(0)=e^{-i \ominus} B \circ A \circ B^{-1}
$$

where $B=\Phi^{\prime}(\zeta)$.

Now if $x \neq 0, x \in \mathcal{B}$ satisfies (41), then $A_{1} z=z$, where $z=B x \in \mathcal{B}$ and $z \neq 0$.

Now again it follows from Rudin's Theorem that

$$
\mathcal{F}_{1}:=F_{i x_{\mathcal{B}}}\left(F_{1}\right)=\operatorname{ker}\left(I-A_{1}\right) \cap \mathcal{B}=\mathcal{N} \neq\{0\} .
$$

Therefore, one can find $z \in \mathcal{N}$, such that $z \notin \Phi(E(\tau, m))$, hence $u=\Phi^{-1}(z) \notin E(\tau, m)$.

We claim that there is $x \in \mathcal{B}$ such that $F(x)=u$. Indeed, set $y=e^{-i \ominus} z$ and $x=\Phi^{-1}(y) \in \mathcal{B}$. Since $z \in \mathcal{N}$ the element $y$ also belongs to $\mathcal{N}$ and we have $F_{1}(y)=y$. Then we get by (42) that

$$
u=\Phi^{-1}(z)=\Phi^{-1}\left(e^{i \ominus} y\right)=\Phi^{-1}\left(e^{i \ominus} F_{1}(y)\right)=F\left(\Phi^{-1}(y)\right)=F(x) .
$$

This contradicts our assumption.

So, we have proved that the whole spectrum $\Sigma(A)$ belongs to $\Delta$.

Since $\Sigma(A)$ is compact, one can find $\varepsilon>0$, such that the spectral radius $r(A)<1-\varepsilon$.

This means that there is a norm $\|\cdot\|_{1}$ in $\mathcal{H}$ equivalent to the original norm of $\mathcal{H}$ such that $\sup _{\|x\|_{1} \leq 1}\|A x\|_{1}<1$ (see, for example, Krasnoselskii, M. A. et al., 1969). Since $A=F^{\prime}(\zeta)$ and $\zeta=F(\zeta)$, this implies by the local uniform continuity of $F^{\prime}(x)$ that there are positive numbers $r>0$ and $\delta>0$ small enough such that

$$
\|F(x)-\zeta\| \leq(1-\delta)\|x-\zeta\|
$$

whenever $\|x-\zeta\| \leq r$.

Therefore, $\left\{F^{n}\right\}_{n=1}^{\infty}$ converge uniformly on the ball $\{x:\|x-\zeta\|<r\} \subset \mathcal{B}$ to the point $\zeta$. Applying Vitali's convergence Theorem (see, for example, Reich, S. et al., 2005) we complete assertion (ii).

Assertions (iii) and (iv) are direct results of Theorems 9 and 10.

\section{Acknowledgment}

The author is very thankful to Prof. Simeon Reich, Prof. Mark Elin, and Dr. Felix Kerdman for useful remarks and discussions. 


\section{References}

Abate, M. (1998). The Julia-Wolff-Carathéodory theorem in polydisks. Journal D'analyse Mathématique, 74, $275-306$. http://dx.doi.org/10.1007/BF02819453

Abate, M., \& Tauraso, R. (1999). The Julia-Wolff-Carathéodory theorem(s). Contemporary Mathematics, 222, $161-172$.

Cowen, C. C., \& MacCluer, B. D. (1995). Composition Operators on Spaces of Analytic Functions. Boca Raton, FL: CRC Press.

Dineen, S. (1989). The Schwarz Lemma. Oxford, UK: Clarendon Press.

Earle, C. J., \& Hamilton, R. S. (1970). A fixed point theorem for holomorphic mappings. Proceedings of Symposia in Pure Mathematics, 16, Amer. Math. Soc., Providence, R. I., 61-65.

Elin, M., Reich, S., \& Shoikhet, D. (2002). Asymptotic behavior of semigroups of $\rho$-nonexpansiveand holomorphic mappings on the Hilbert ball. Annali di Matematica Pura ed Applicata, 181, 501-526. http://dx.doi.org/10.1007/s10231-002-0052-2

Franzoni, T., \& Vesentini, E. (1980). Holomorphic Maps and Invariant Distances. Amsterdam, North-Holland: NorthHolland Publishing Company.

Goebel, K., \& Reich, S. (1982). Iterating holomorphic self-mappings of the Hilbert ball. Proceedings of the Japan Academy, 58, 349-352. http://dx.doi.org/10.3792/pjaa.58.349

Goebel, K., \& Reich, S. (1984). Uniform Convexity, Hyperbolic Geometry and Nonexpansive Mappings. New York and Basel: Marcel Dekker.

Goebel, K., Sekowski, T., \& Stachura, A. (1980). Uniform convexity of the hyperbolic metric and fixed points of holomorphic mappings in the Hilbert ball. Nonlinear Analysis, 4, 1011-1021. http://dx.doi.org/10.1016/0362-546X(80)90012-7

Harris, L. A. (2003). Fixed points of holomorphic mappings for domains in Banach spaces. Abstract and Applied Analysis, 5, 261-274. http://dx.doi.org/10.1155/S1085337503205042

Hervé, M. (1963). Several Complex Variables: Local Theory. London: Tata Institute of Fundamental Research, Bombay Oxford University Press.

Hervé, M. (1989). Analyticity in Infinite Dimensional Spaces. Berlin, Germany: Walter de Gruyter.

Kaashek, M. A., \& West, T. T. (1968). Locally compact monothetic semi-algebras. Proceedings of the London Mathematical Society, 18, 428-438.

Kapeluszny, J., Kuczumow, T., \& Reich, S. (1999). The Denjoy-Wolff theorem in the open unit ball of a strictly convex Banach space. Advances in Mathematics, 143, 111-123. http://dx.doi.org/10.1155/S1085337503205042

Kapeluszny, J., Kuczumow, T., \& Reich, S. (1999). The Denjoy-Wolff theorem for condensing holomorphic mappings. Journal of Functional Analysis, 167, 79-93. http://dx.doi.org/10.1006/jfan.1999.3448

Khatskevich, V., Reich, S., \& Shoikhet, D. (1995). Fixed point theorems for holomorphic mappings and operator theory in indefinite metric spaces. Integral Equations and Operator Theory, 22, 305-316. http://dx.doi.org/10.1007/BF01378779

Kopecká, E., \& Reich, S. (2009). Asymptotic behavior of resolvents of coaccretive operators in the Hilbert ball. Nonlinear Analysis, 70, 3187-3194. http://dx.doi.org/10.1016/j.na.2008.04.023

Krasnoselskii, M. A., Vainikko, G. M., Zabreiko, P. P., Ruticki, Ya. B., \& Stecenko, V. Ya. (1969). Approximate Solution of Operator Equations. Moscow: Nauka.

Kuczumow, T., Reich, S., \& Shoikhet, D. (2001). Fixed points of holomorphic mappings: A metric approach. In W. A. Kirk, \& B. Sims, (Eds.), Handbook of Metric Fixed Point Theory, pp. 437-515. Dordrecht, Holland: Kluwer.

Lyubich, Yu., \& Zemánek, J. (1994). Precompactness in the uniform ergodic theory. Studia Mathematica, 112 (1), 89-97.

MacCluer, B. D. (1983). Iterates of holomorphic self-maps of the unit ball in $\mathbb{C}^{n}$. The Michigan Mathematical Journal, 30, 97-106.

Reich, S. (1985). Averaged mappings in the Hilbert ball. The Journal of Mathematical Analysis and Applications, 109, 199-206. http://dx.doi.org/10.1016/0022-247X(85)90187-8

Reich, S. (1993). The alternating algorithm of von Neumann in the Hilbert ball. Dynamic Systems \& Applications, 2, 21-25. 
Reich, S., \& Shoikhet, D. (1997). The Denjoy-Wolff theorem. Annales Universitatis Mariae Curie-Sklodowska, 51, 219-240.

Reich, S., \& Shoikhet, D. (2002). The Denjoy-Wolff Theorem. Math. Encyclopaedia, Supplement 3, Kluwer Academic Publishers, 121-123.

Reich, S., \& Shoikhet, D. (2005). Nonlinear Semigroups, Fixed Points, and Geometry of Domains in Banach Spaces. London: Imperial College Press.

Rudin, W. (1980). Function Theory on the Unit Ball in $\mathbb{C}^{n}$. Berlin, Germany: Springer.

Shapiro, J. H. (1993). Composition Operators and Classical Function Theory. Berlin, Germany: Springer.

Shoikhet, D. (2001). Semigroups in Geometrical Function Theory. Dordrecht, Holland: Kluwer.

Stachura, A. (1985). Iterates of holomorphic self-maps of the unit ball in Hilbert spaces. Proceedings of the American Mathematical Society, 93, 88-90.

Święch, A. (1991). Spectral characterization of operators with precompact orbit. Studia Mathematica, 96, $277-282$.

Vesentini, E. (1985). Iterates of holomorphic mappings. Uspekhi Matematicheskikh Nauk, 40, 13-16. 